\title{
Metabolic Syndrome as a Risk Factor for Barrett's Esophagus: A Population Based Case Control Study
}

\author{
Cadman L. Leggett, MD11, Eric M. Nelsen, MD², Jianmin Tian ${ }^{1}$, Cathy Schleck ${ }^{3}$, Alan R. \\ Zinsmeister, PhD $^{3}$, Kelly Dunagan, RN ${ }^{1}$, G. Richard Locke, MD ${ }^{1}$, Kenneth K. Wang, MD ${ }^{1}$, \\ Nicholas J. Talley, MD ${ }^{1,4}$, and Prasad G. Iyer, MD, MS ${ }^{1}$ \\ ${ }^{1}$ Division of Gastroenterology and Hepatology, Mayo Clinic, Rochester MN \\ ${ }^{2}$ Department of Internal Medicine, Mayo Clinic, Rochester MN \\ ${ }^{3}$ Division of Biomedical Statistics and Informatics, Mayo Clinic, Rochester MN \\ ${ }^{4}$ Faculty of Health, The University of Newcastle, Australia
}

\begin{abstract}
Objective-To assess the association between Barrett's esophagus (BE) and the Metabolic Syndrome in subjects with and without reflux symptoms and to determine if this association is reflux independent and metabolically driven.
\end{abstract}

Patients and Methods-BE cases and controls were residents of Olmsted County, MN (19992006). Two control groups (one with and one without symptoms of gastroesophageal reflux) were identified from a cohort of subjects who had responded to a validated GI symptom questionnaire. Cases and controls were individually matched by age, sex and duration of follow-up. Controls did not have a known diagnosis of BE. The association of the Metabolic Syndrome and its individual components with $\mathrm{BE}$ was assessed using univariate and multiple variable conditional logistic regression separately for each control group.

Results-309 subjects were included (103 BE cases, 103 controls with reflux symptoms and 103 controls without reflux symptoms). $64 \%$ of cases, $47 \%$ of controls with reflux symptoms and $50 \%$ of controls without reflux symptoms had the Metabolic Syndrome. The Metabolic Syndrome was associated with a twofold increased risk of $\mathrm{BE}$ relative to subjects with $(\mathrm{OR}=2.00(1.10,3.65)$ $\mathrm{p}=0.02)$ and without $(\mathrm{OR}=1.90(1.03,3.60), \mathrm{p}=0.04)$ reflux symptoms. This association was independent of smoking, alcohol consumption and BMI and remained robust with sensitivity analysis.

Conclusions-The Metabolic Syndrome is associated with BE independent of reflux symptoms. This may reflect a reflux independent pathway of BE pathogenesis.

Impact: Subjects with the Metabolic syndrome may be at higher risk for BE and esophageal adenocarcinoma.

(C) 2012 Mayo Foundation for Medical Education and Research. Published by Elsevier Inc. All rights reserved.

CORRESPONDING AUTHOR: Prasad G. Iyer, MD MS, Division of Gastroenterology and Hepatology, 200 1st St SW, Rochester, MN 55905 iyer.prasad@ mayo.edu, Tel: 5072556930, Fax: 5072557612.

Publisher's Disclaimer: This is a PDF file of an unedited manuscript that has been accepted for publication. As a service to our customers we are providing this early version of the manuscript. The manuscript will undergo copyediting, typesetting, and review of the resulting proof before it is published in its final citable form. Please note that during the production process errors may be discovered which could affect the content, and all legal disclaimers that apply to the journal pertain.

FINANCIAL SUPPORT AND DISCLOSURE:

Supported by a Junior Faculty Development Award from the American College of Gastroenterology, the NIDDK (RC4DK090413) and the Mayo Foundation. 


\section{Keywords}

arrett's Esophagus; Metabolic syndrome; Obesity; Visceral fat; Screening; Esophageal Adenocarcinoma

\section{Objective}

The incidence of obesity and its associated morbidity and mortality continue to rise in developed nations ${ }^{1}$. Epidemiological studies suggest that central obesity and associated increased abdominal visceral fat is a risk factor for several types of cancers, including esophageal adenocarcinoma (EA) ${ }^{2,3}$. Multiple mechanisms have been implicated to account for this observation. Elevated intra-abdominal pressure in centrally obese subjects may worsen gastroesophageal reflux through mechanical disruption of the gastroesophageal junction leading to esophageal injury and development of Barrett's esophagus (BE), the precursor lesion for $\mathrm{EA}^{4}$. Abdominal adipose tissue, especially visceral fat, is also considered to be metabolically active secreting inflammatory mediators, cytokines and insulin-like growth factors, leading to a systemic inflammatory and insulin resistant state which is a fertile environment for carcinogenesis ${ }^{5}$. There is evidence to suggest that the Metabolic syndrome is a direct risk for gastrointestinal and hepatic carcinomas ${ }^{6}{ }^{9}$, suggesting an obesity mediated reflux independent pathway of promoting carcinogenesis. It has been hypothesized that this heightened state of inflammation and insulin resistance may predispose to esophageal carcinogenesis ${ }^{10,11}$.

The association between obesity, as measured by BMI and BE has been somewhat inconsistent with a meta-analysis concluding that this is mediated by increasing reflux, with only population based controls having greater BMI than BE cases and not reflux controls ${ }^{12}$. Subsequent studies found that BE is associated with abdominal obesity (measured by waist circumference or waist hip ratio) independent of BMI in epidemiologic studies ${ }^{4,13}$. This led to the hypothesis that fat distribution and not overall adiposity is responsible for elevated cancer risk. We have previously reported the association of $\mathrm{BE}$, esophageal inflammation and dysplasia with increased visceral fat, suggesting a reflux independent mechanism for BE pathogenesis ${ }^{10,14}$. Studies have also demonstrated the influence of visceral fat and the Metabolic syndrome on esophageal inflammation ${ }^{15,16}$. However, there is no data on the association of the Metabolic syndrome and its associated components (central obesity, diabetes mellitus type II (DM2), hypertension (HTN), hyperlipidemia) which reflect the systemic consequence of central obesity, with BE. Moreover, it is also unclear if this association is mediated through reflux or is independent of reflux. Association with the Metabolic syndrome and its individual components, particularly independent of reflux would be crucial in supporting the hypothesis that central obesity (via increased visceral fat and visceral fat produced mediators) may have a reflux independent role in mediating esophageal metaplasia and carcinogenesis.

Identification of high-risk groups for developing EA is important in developing and targeting early detection strategies. Given the asymptomatic nature of BE, screening is the only method of identifying individuals with this disease. The purpose of this case-control study was to assess the association of the Metabolic syndrome, both as a composite diagnosis and its individual components, with BE to test our hypothesis that the Metabolic syndrome (and by extension, the insulin resistance and systemic inflammatory milieu in these subjects) plays a reflux independent role in the pathogenesis of esophageal metaplasia. Towards this end, in this study design we incorporated control groups with and without reflux symptoms (population controls) to additionally assess the role of reflux in the association of $\mathrm{BE}$ and the Metabolic syndrome. 


\section{PATIENTS AND METHODS}

This study was approved by the Mayo Clinic and the Olmsted Medical Center Institutional Review Boards.

\section{The Rochester Epidemiology Project}

The Rochester Epidemiology Project (REP) is a searchable medical record linkage system for the population of Olmsted Country, $\mathrm{MN}^{17}$. This population is comprised of $\sim 120,000$ persons, of whom $89 \%$ are non-hispanic white; socio-demographically the community is similar to the US non-hispanic white population. Annually, most REP participants seek medical care within the Mayo Medical Center, Olmsted Medical Center, Rochester Family Medicine Clinic and/or other medical care providers in Olmsted County. As of July 2009, the REP database contained information on over 400,000 individuals. This allows access to the full medical records of all subjects in Olmsted County, MN regardless of where they seek medical care.

\section{Subject Definition}

Sampling frame-Consisted of population based (Olmsted County) cohorts for both cases and controls. Figure 1 summarizes the process of case and control selection.

BE cases-This has been described previously ${ }^{18}$. Briefly, a total of 401 cases were identified from Olmsted County using the REP through an electronic search using the International Classification of Disease, $9^{\text {th }}$ version (ICD-9) code for BE (530.85) which provided a sensitive search, followed by a chart review, to increase specificity. Inclusion criteria included: BE cases (endoscopic visualization of columnar epithelium and histopathological confirmation of intestinal metaplasia) alive as of 5/7/2010, those diagnosed between 1999 to 2006 (to facilitate abstraction from electronic medical records which are available from 1996 onwards) and those providing research authorization. Endoscopic and histopathologic reports from each esophagogastroduodenoscopy (EGD) were reviewed. All endoscopic examinations were performed by board-certified gastroenterologists on staff at the Mayo Clinic or Olmsted Medical Center.

Controls-Controls were identified via random selection from a cohort of Olmsted County residents who responded to a validated revised Talley-Bowel Disease Questionnaire administered in $2008^{19}$. The questionnaire had been mailed to an age and sex stratified random sample of Olmsted county residents and included questions on the frequency and nature of gastroesophageal reflux symptoms including heartburn and reflux. Questions on gastroesophageal reflux included: How often have you had heartburn, a burning or ache behind the breast bone in the chest? (do not count pain from angina or heart trouble); How often have you noticed a very sour or acid-tasting fluid at the back of your throat. Response options included: Never, <1 day a month, 1 day a month, 2 or 3 days a month, 1 day a week, $>1$ day a week, every day.

Two non-overlapping control groups were selected (figure 2). The first control group consisted of 404 individuals who reported gastroesophageal reflux symptoms at least once a week on the questionnaire. The second control group was drawn from 3,111 individuals who did not report any gastroesophageal reflux symptoms on the questionnaire (population controls). All controls did not have a known diagnosis of BE in their comprehensive medical record as determined by an ICD9 code (530.85 for Barrett's Esophagus) search in the diagnostic index for each patient, review of endoscopy reports and detailed review of the medical records. This is consistent with the methodology of prior studies 4,20 
Each case was then individually matched to a control from each control group based on age ( $\pm 10 \mathrm{yrs}$ ), sex and duration of follow-up as given by date of first registration at Mayo. Matching on race was not done given that more than $95 \%$ of cases and controls were nonhispanic white. Potential participants were excluded if they were deceased at the time of the start of the study or if they had not provided research authorization.

\section{Data Collection}

Medical records were independently reviewed by one of the investigators. Information was abstracted for every subject within each matched set. The date of diagnosis of BE ( \pm 1 year) for case subjects served as a cross sectional reference date in both control groups. Demographic information (age, sex, ethnicity) was abstracted for all study participants. History of tobacco and alcohol consumption was recorded at the cross sectional reference date. Anthropometric measurements (weight, height) were abstracted at both the cross sectional reference date and last medical encounter. Each subject's medical and medication history was reviewed at the cross sectional reference date (within 1 year of BE diagnosis). The use of medications to treat DM2, HTN, and hyperlipidemia as well as the use of aspirin, non-steroidal anti-inflammatory drugs, proton-pump inhibitors and histamine-2 receptor blockers were recorded for each time interval. Complete medical records for all cases and controls were available for review given resources of the REP which links all medical records at all medical providers within Olmsted County. The date of diagnosis of the Metabolic syndrome risk factors (DM2, HTN, hyperlipidemia) was obtained from a review of the participant's primary care physician's problem list and/or medication history. Supportive laboratory data (fasting blood glucose, hemoglobin A1C, fasting lipid panel) and blood pressure readings were recorded at the cross sectional reference date and at the most recently available visit. A diagnosis of Metabolic syndrome was made only if criteria for their diagnosis were met within the cross sectional reference date range for cases (within one year of BE diagnosis) and controls.

Each participant's endoscopic history was reviewed. Information on number of EGDs, date of procedure, indication, findings (hiatal hernia, reflux esophagitis, eosinophilic esophagitis, nodules/masses, stricture/rings) and site of biopsy, if taken (esophagus, esophagogastric junction, stomach) was recorded. Erosive esophagitis was defined using the Los Angeles classification (grades A-D) ${ }^{21}$. Histopathology reports were reviewed for evidence of intestinal metaplasia by a gastrointestinal pathologist.

\section{Definition of the Metabolic syndrome}

Our study definition for the Metabolic syndrome included the presence of two or more of the following: BMI >30, DM2, HTN, hyperlipidemia ${ }^{22}$. BMI was calculated from measurements of weight and height using the formula: weight $(\mathrm{kg})$ divided by the square of height $(\mathrm{m})$ within a year of the reference cross sectional date. Diagnosis of the Metabolic syndrome components was established by the subject's primary care physician diagnoses (as listed in the medical problem list) and/or the patient's medication history within a year of the reference date. Given the unavailability of anthropometric measurements (waist circumference or waist hip ratio) in the medical record, we used BMI> 30 as a component of the diagnostic criteria for the Metabolic syndrome. A BMI > 30 correlates well with increased waist circumference (abdominal obesity) and has also been used as a criterion for the diagnosis of Metabolic syndrome by the World Health Organization ${ }^{23}$ and the International Diabetes Federation ${ }^{24}$.

\section{Statistical Analyses}

The associations of the specific Metabolic syndrome components and overall Metabolic syndrome status (no vs. yes) with BE were defined a priori and assessed using conditional 
logistic regression models separately for each control group. Univariate odds ratios (OR) and $95 \%$ confidence intervals (CIs) were computed using the estimated model coefficients and their standard errors. A separate multiple variable model for each control group was also examined incorporating the individual Metabolic syndrome components as predictor variables. The multiple variable models were limited to those matched pairs with complete data. The potential selection bias resulting from restriction to matched pairs with complete data was checked using an unconditional logistic regression model (subject included vs. not included as the binary dependent variable and specific demographic features, e.g. age and sex, along with case and reflux positive/negative control status as predictors).

Mitigation of possible misclassification bias: There is the potential for some of the controls to have BE since all controls did not undergo an endoscopy to confirm absence of BE. An assessment of the sensitivity of the analyses to this potential misclassification bias was examined by repeated (100 times) random re-assignment of some of the controls (2\%-10\%) in each control group (separately, depending on their MetS status and GER symptom status) as "cases".

\section{Sample Size}

With 100 matched pairs, there would have been approximately $80 \%$ power (2-sided alpha level of 0.05 ) to detect an odds ratio of 2.25 assuming the proportion of controls with the Metabolic syndrome was $45-50 \%$ and the matching did not induce within pair correlations in risk factor exposure. Assuming a modest amount of correlation induced by the matching, there would have been approximately $80 \%$ power to detect an odds ratio of 2.75 , where again the proportion of controls with the Metabolic syndrome was 45-50\%.

\section{RESULTS}

\section{Baseline Characteristics}

A total of 309 subjects were included in the study (103 BE cases, 103 controls with reflux symptoms, 103 controls without reflux symptoms). 45 (44\%) of controls with reflux and 23 (22\%) of controls without reflux underwent endoscopy to confirm the absence of BE. Erosive esophagitis was present in $7 \%$ of controls with reflux symptoms (grade A, $\mathrm{n}=4$; grade $\mathrm{B}, \mathrm{n}=1$; unclassified, $\mathrm{n}=2$ ) and $3 \%$ of controls without reflux symptoms (grade $\mathrm{A}$, $\mathrm{n}=3$ ). The mean (SD) length of the BE segment in cases was $4 \mathrm{~cm} \mathrm{(3).} \mathrm{Overall,} 86$ (84\%) of BE cases had no dysplasia, 17 (16\%) of cases had low-grade dysplasia and none had highgrade dysplasia at initial diagnosis.

Baseline characteristics of participants are summarized in table 1. Cases and controls were individually matched by age, sex and duration of follow up and had similar characteristics except for more BE cases using PPIs (53\%) compared to controls with (15\%) and without $(5 \%)$ reflux symptoms. A total of $64 \%$ of cases, $47 \%$ of controls with reflux symptoms and $50 \%$ of controls without reflux symptoms met diagnostic criteria for the Metabolic syndrome.

\section{Association between Metabolic syndrome and Barrett's Esophagus}

Univariate analysis between the Metabolic syndrome (both as a composite diagnosis and its individual components) and $\mathrm{BE}$ is summarized in table2. A total of 98 matched pairs were used for this model. Five pairs were excluded due to missing data. The Metabolic syndrome as a composite diagnosis was associated with an approximately twofold increased risk of $\mathrm{BE}$ relative to controls with $(\mathrm{OR}=2.00(1.10,3.65) \mathrm{p}=0.02)$ and without $(\mathrm{OR}=1.90(1.03,3.60)$, $\mathrm{p}=0.04$ ) reflux symptoms. No association of BE was seen with smoking and heavy alcohol intake on univariate analysis (see table 2). Univariate analysis with ethnicity as a variable 
provided unstable odds ratio estimates likely due to the high (>95\%) proportion of individuals of non-hispanic white race in all three groups. Univariate analysis for each of the individual Metabolic syndrome components demonstrated a positive association between $\mathrm{HTN}$ and BE in subjects without reflux symptoms $(\mathrm{OR}=2.36(1.17,4.78), \mathrm{p}=0.02)$.

A multiple variable model was examined to assess the association of BE with the complete set of individual components of the Metabolic syndrome. (table 3) The odds ratio for each component was thus adjusted for the remaining components. No significant associations between the individual components of the Metabolic syndrome and BE were found using this multiple variable model, though HTN remained statistically significant in the model using reflux negative controls. Thus, although the estimated odds ratio for HTN remained the same in the multiple variable model as in the corresponding univariate model, the reduced sample size (i.e. number of matched pairs) in the multiple variable resulted in a less precise estimate (i.e. wider $\mathrm{CI}$ ).

The multiple variable models had a total of 81 matched pairs for the control group with reflux symptoms and 83 pairs for the control group without reflux symptoms. The primary reason for the reduction in matched pairs could be attributed to missing values for BMI in close proximity (within a year) of the cross sectional reference date (diagnosis date for BE case) for the case or its matched control. Logistic regression to predict a missing BMI value using age, sex, the remaining components of the Metabolic syndrome definition, and subject status (case as the reference level, controls with reflux, controls without reflux) indicated that controls with reflux had an increased odds to be associated with a missing BMI value.

The length of the Barrett's segment was only associated with BMI (Spearman $r=-0.24$ $[\mathrm{p}=0.015], \mathrm{n}=100$ ) but not with the Metabolic syndrome or its components.

\section{Mitigation of misclassification bias (quasi sensitivity analysis)}

The average (over the 100 replications) point estimates (i.e. Odds Ratios) for the univariate association of Metabolic syndrome with BE were virtually identical to those obtained from the complete data: $2.0(1.8,2.4)$ for reflux controls, and $1.8(1.6,2.0)$ for non-reflux controls. The intervals using $5^{\text {th }}$ and $95^{\text {th }}$ percentiles of the distribution of repeated Odds Ratios were somewhat tighter than the estimated $95 \%$ CIs from the complete data analyses.

\section{Discussion}

We performed a population based case-control study to assess the association of BE and the Metabolic syndrome. Our analysis suggests that individuals with the Metabolic syndrome are at an approximately twofold increased risk of BE. We included controls with reflux symptoms and controls without reflux symptoms (population based controls) in this study, allowing us to study the influence of reflux on this association. We found that this association is independent of reflux symptoms given consistent results in both controls with and without reflux symptoms. The association appeared to be stronger in reflux controls. Individual components of the Metabolic syndrome did not appear to be associated with BE in this analysis, suggesting perhaps that the syndrome as a whole and its associated state of metabolic derangement may contribute to BE pathogenesis.

The Metabolic syndrome has been defined by several institutions including the World Health Organization, National Cholesterol Education Program's Adults Treatment Panel and the International Diabetes Federation. Published definitions differ in terms of criteria and are limited by poorly defined thresholds ${ }^{25}$. In our study, we utilize a Metabolic syndrome definition with established end-points of central obesity, hypertension, hyperlipidemia and diabetes mellitus type II that allowed for more precise retrospective abstraction of data. 
Given the lack of anthropometric measurements in medical records, we defined central obesity as $\mathrm{BMI}>30$ as per criteria in the $\mathrm{WHO}^{23}$ and International Diabetes Federation definitions of the Metabolic syndrome ${ }^{24}$, as $>95 \%$ of these individuals will have a waist circumference greater than sex and ethnic specific threshold values used to define Metabolic syndrome. Previous epidemiological studies within the Olmsted County population have established the prevalence of the Metabolic syndrome to be approximately $48 \%$ in older individuals using the International Diabetes Federation criteria ${ }^{26}$. This prevalence is similar to our control groups (47\% of controls with reflux symptoms and $50 \%$ of controls without reflux symptoms) using our definition, further validating the findings in this study and reducing the possibility of misclassification bias for Metabolic syndrome. The prevalence of the Metabolic syndrome has been shown to be higher in patients with BE in prior studies and these estimates are also similar to our findings ${ }^{3}$.

Cross-sectional case control and prospective studies have shown that the Metabolic syndrome is a predisposing factor for reflux esophagitis, a risk factor for progression of esophagitis influenced by increased abdominal and visceral fat ${ }^{15,27}$. Additional studies have found a strong association between esophagitis and visceral abdominal fat but not abdominal subcutaneous fat ${ }^{16}$. Our findings suggest that the Metabolic syndrome, by creating a proinflammatory, insulin resistant milieu may promote esophageal injury, metaplasia and neoplasia, independent of reflux. This distinguishes our study from previously published data suggesting an association between $\mathrm{BE}$ and measures of abdominal obesity. The prevalence of asymptomatic esophagitis in the Metabolic syndrome population is not known, making it hard to determine the role that reflux plays in disease compared to other potential risk factors.

The Metabolic syndrome is associated with central obesity and increased visceral fat ${ }^{28}$. Visceral fat is metabolically active and known to secrete insulin-like growth factors, adipocytokines (leptin, adiponectin) and inflammatory cytokines (tumor necrosis factor alpha, interleukins) ${ }^{29}$. These peptides can directly and indirectly promote cell proliferation and angiogenesis as well as inhibit cellular apoptosis ${ }^{29}$. Several cellular pathways involving these peptides have been implicated in BE and EA pathogenesis ${ }^{30}$. Emerging evidence seems to suggest a prominent role for the insulin-insulin like growth factor axis in the obesity esophageal carcinogenesis pathway ${ }^{11,31-33}$ : this mechanism has been implicated in other organs such as the colon ${ }^{9}$, breast and prostate.

Given the rising incidence of obesity in developed nations it is important to establish which subset of individuals is at higher risk of BE in order to potentially perform cost-effective screening for esophageal adenocarcinoma. Our study suggests that individuals with the Metabolic syndrome may be at a higher risk of developing BE even in the absence of reflux symptoms. Prospective studies are needed to substantiate our findings.

Our study is limited by the lack of anthropometric measurements (waist circumference or waist hip ratio) to define the Metabolic syndrome. Previous studies looking at the correlation of BMI and waist circumference, however, have demonstrated that BMI (especially greater than 30 ) is an appropriate predictor of visceral adipose tissue in both sexes ${ }^{28}$. This has led the International Diabetes Federation and the WHO definitions of the Metabolic syndrome to use BMI>30 as a threshold for central obesity. Another potential limitation of our study is that absence of BE was confirmed by endoscopy in $44 \%$ of reflux controls and $22 \%$ of population controls, raising the possibility of misclassification bias. However, any misclassification bias in the controls would likely bias our results towards the null (i.e. against the hypothesis), and make our estimates more conservative and less likely to be due to a type 1 error. The population prevalence of $\mathrm{BE}$ has been reported to be $1-2 \%$ in European studies ${ }^{34}$. Furthermore, recognizing the possibility of misclassification bias due to 
the lack of endoscopy in all controls, we randomly reassigned 1-2\% of reflux negative controls and 5-10\% of reflux positive controls as cases (see method section and results) and found that the association estimates of BE with Metabolic syndrome remained robust. Though retrospective, given the lack of a standardized tool to assess reflux symptomatology in the primary care setting, we identified our control groups with the use of a validated gastrointestinal questionnaire. While the population studied in this study was predominantly older, non-hispanic white males with increased BMI, the incidence of BE and esophageal adenocarcinoma is known to be higher in a population with these characteristics allowing us to reach statistical power for our analysis. Moreover, the study population formed part of an established clinical database that allowed us to perform a detailed retrospective review with low likelihood of missing data on confounding variables. There currently is no available data on the Metabolic Syndrome and BE in non-white populations to determine if our results can be extrapolated to other populations. Our study also draws its strength from the population based, comprehensive matching of cases and controls in an attempt to factor out $\mathrm{BE}$ confounders. Erosive esophagitis may lead to BE and may be present in asymptomatic individuals. In our study, $7 \%$ of controls with reflux symptoms and 3\% of controls without reflux symptoms who had undergone an upper endoscopy were found to have mild erosive esophagitis. The estimated prevalence of asymptomatic erosive esophagitis in our study cohort, however, is below that of the general population were it is calculated to be around $26.4 \%$ to $45.3 \%^{35,36}$.

\section{Conclusion}

Our findings suggest that individuals with the Metabolic syndrome are at a near twofold increase risk of BE. If confirmed by prospective studies, subject with the Metabolic syndrome may constitute a high risk group for BE and esophageal adenocarcinoma. With a rising incidence of obesity and the Metabolic syndrome, this population may also benefit from preventive therapy. More insight is needed into the pathophysiology of $\mathrm{BE}$ and the role of visceral fat and whether weight loss reverses the risk of BE in the obese.

\section{Supplementary Material}

Refer to Web version on PubMed Central for supplementary material.

\section{Acknowledgments}

We appreciate the assistance provided by Dr. Gary Falk in reviewing the manuscript.

\section{ABBREVIATIONS}

$\begin{array}{ll}\text { BE } & \text { Barrett's Esophagus } \\ \text { EA } & \text { Esophageal Adenocarcinoma } \\ \text { DM2 } & \text { Diabetes Mellitus Type II } \\ \text { HTN } & \text { Hypertension } \\ \text { REP } & \text { Rochester Epidemiology Project } \\ \text { ICD-9 } & \text { International Classification of Disease, } 9^{\text {th }} \text { Version } \\ \text { EGD } & \text { Esophagogastroduodenoscopy } \\ \text { OR } & \text { Odds Ratios } \\ \text { CIs } & \text { Confidence Intervals }\end{array}$




\section{REFERENCES}

1. Flegal KM, Carroll MD, Ogden CL, Curtin LR. Prevalence and trends in obesity among US adults, 1999-2008. JAMA. 2010; 303:235-241. [PubMed: 20071471]

2. Corley DA, Kubo A, Zhao W. Abdominal obesity and the risk of esophageal and gastric cardia carcinomas. Cancer Epidemiol Biomarkers Prev. 2008; 17:352-358. [PubMed: 18268119]

3. Ryan AM, Healy LA, Power DG, et al. Barrett esophagus: prevalence of central adiposity, metabolic syndrome, and a proinflammatory state. Ann Surg. 2008; 247:909-915. [PubMed: 18520215]

4. Corley DA, Kubo A, Levin TR, et al. Abdominal obesity and body mass index as risk factors for Barrett's esophagus. Gastroenterology. 2007; 133:34-41. quiz 311. [PubMed: 17631128]

5. Russo A, Autelitano M, Bisanti L. Metabolic syndrome and cancer risk. Eur J Cancer. 2008; 44:293-297. [PubMed: 18055193]

6. Welzel TM, Graubard BI, Zeuzem S, El-Serag HB, Davila JA, McGlynn KA. Metabolic syndrome increases the risk of primary liver cancer in the United States: a study in the SEER-Medicare database. Hepatology. 2011; 54:463-471. [PubMed: 21538440]

7. Rosato V, Tavani A, Bosetti C, et al. Metabolic syndrome and pancreatic cancer risk: a case-control study in Italy and meta-analysis. Metabolism. 2011; 60:1372-1378. [PubMed: 21550085]

8. Pelucchi C, Negri E, Talamini R, et al. Metabolic syndrome is associated with colorectal cancer in men. Eur J Cancer. 2010; 46:1866-1872. [PubMed: 20395126]

9. Ortiz AP, Thompson CL, Chak A, Berger NA, Li L. Insulin resistance, central obesity, and risk of colorectal adenomas. Cancer. 2011

10. El-Serag HB, Kvapil P, Hacken-Bitar J, Kramer JR. Abdominal obesity and the risk of Barrett's esophagus. Am J Gastroenterol. 2005; 100:2151-2156. [PubMed: 16181362]

11. Greer KB, Thompson CL, Brenner L, et al. Association of insulin and insulin-like growth factors with Barrett's oesophagus. Gut. 2011

12. Cook MB, Greenwood DC, Hardie LJ, Wild CP, Forman D. A systematic review and metaanalysis of the risk of increasing adiposity on Barrett's esophagus. Am J Gastroenterol. 2008; 103:292-300. [PubMed: 17986313]

13. Edelstein ZR, Farrow DC, Bronner MP, Rosen SN, Vaughan TL. Central adiposity and risk of Barrett's esophagus. Gastroenterology. 2007; 133:403-411. [PubMed: 17681161]

14. Nelsen EMKY, Takahashi N, Shi Q, Lewis JT, Namasivayam V, Buttar NS, Dunagan KT, Prasad GA. Distribution of Body Fat and its Influence on Esophageal Inflammation and Dysplasia in Patients with Barrett's Esophagus. Clinical Gastroenterology and Hepatology. 2012 Accepted for publication.

15. Chung SJ, Kim D, Park MJ, et al. Metabolic syndrome and visceral obesity as risk factors for reflux oesophagitis: a cross-sectional case-control study of 7078 Koreans undergoing health check-ups. Gut. 2008; 57:1360-1365. [PubMed: 18441006]

16. Nam SY, Choi IJ, Ryu KH, Park BJ, Kim HB, Nam BH. Abdominal visceral adipose tissue volume is associated with increased risk of erosive esophagitis in men and women. Gastroenterology. 2010; 139:1902-1911. e1902. [PubMed: 20727886]

17. St Sauver JL, Grossardt BR, Yawn BP, Melton LJ 3rd, Rocca WA. Use of a medical records linkage system to enumerate a dynamic population over time: the Rochester epidemiology project. Am J Epidemiol. 2011; 173:1059-1068. [PubMed: 21430193]

18. Jung KW, Talley NJ, Romero Y, et al. Epidemiology and natural history of intestinal metaplasia of the gastroesophageal junction and Barrett's esophagus: a population-based study. Am J Gastroenterol. 2011; 106:1447-1455. quiz 1456. [PubMed: 21483461]

19. Rey E, Locke GR 3rd, Jung HK, et al. Measurement of abdominal symptoms by validated questionnaire: a 3-month recall timeframe as recommended by Rome III is not superior to a 1-year recall timeframe. Aliment Pharmacol Ther. 2010; 31:1237-1247. [PubMed: 20222912]

20. Kubo A, Levin TR, Block G, et al. Alcohol types and sociodemographic characteristics as risk factors for Barrett's esophagus. Gastroenterology. 2009; 136:806-815. [PubMed: 19111726]

21. Armstrong D, Bennett JR, Blum AL, et al. The endoscopic assessment of esophagitis: a progress report on observer agreement. Gastroenterology. 1996; 111:85-92. [PubMed: 8698230] 
22. Grundy SM, Brewer HB Jr. Cleeman JI, Smith SC Jr, Lenfant C. Definition of metabolic syndrome: report of the National Heart, Lung, and Blood Institute/American Heart Association conference on scientific issues related to definition. Arterioscler Thromb Vasc Biol. 2004; 24:e13e18. [PubMed: 14766739]

23. Consultation. W. Definition. diagnosis classification of diabetes mellitus and its complications. Geneva: World Health Organization; 1999. p. 31-33.1999

24. Alberti KG, Zimmet P, Shaw J. Metabolic syndrome--a new world-wide definition. A Consensus Statement from the International Diabetes Federation. Diabet Med. 2006; 23:469-480. [PubMed: 16681555]

25. Kahn R, Buse J, Ferrannini E, Stern M. The metabolic syndrome: time for a critical appraisal: joint statement from the American Diabetes Association and the European Association for the Study of Diabetes. Diabetes Care. 2005; 28:2289-2304. [PubMed: 16123508]

26. Roberts RO, Geda YE, Knopman DS, et al. Metabolic syndrome, inflammation, and nonamnestic mild cognitive impairment in older persons: a population-based study. Alzheimer Dis Assoc Disord. 2010; 24:11-18. [PubMed: 19568151]

27. Lee YC, Yen AM, Tai JJ, et al. The effect of metabolic risk factors on the natural course of gastrooesophageal reflux disease. Gut. 2009; 58:174-181. [PubMed: 18936105]

28. Oka R, Miura K, Sakurai M, et al. Comparison of waist circumference with body mass index for predicting abdominal adipose tissue. Diabetes Res Clin Pract. 2009; 83:100-105. [PubMed: 19019478]

29. Ryan AM, Duong M, Healy L, et al. Obesity, metabolic syndrome and esophageal adenocarcinoma: epidemiology, etiology and new targets. Cancer Epidemiol. 2011; 35:309-319. [PubMed: 21470937]

30. Kendall BJ, Macdonald GA, Hayward NK, et al. Leptin and the risk of Barrett's oesophagus. Gut. 2008; 57:448-454. [PubMed: 18178609]

31. McElholm AR, McKnight AJ, Patterson CC, Johnston BT, Hardie LJ, Murray LJ. A populationbased study of IGF axis polymorphisms and the esophageal inflammation, metaplasia, adenocarcinoma sequence. Gastroenterology. 2010; 139:204-212. e203. [PubMed: 20403354]

32. Donohoe CL, Doyle SL, McGarrigle S, et al. Role of the insulin-like growth factor 1 axis and visceral adiposity in oesophageal adenocarcinoma. Br J Surg. 2012; 99:387-396. [PubMed: 22241325]

33. Doyle SL, Donohoe CL, Finn SP, et al. IGF-1 and its receptor in esophageal cancer: association with adenocarcinoma and visceral obesity. Am J Gastroenterol. 2012; 107:196-204. [PubMed: 22146489]

34. Ronkainen J, Aro P, Storskrubb T, et al. Prevalence of Barrett's esophagus in the general population: an endoscopic study. Gastroenterology. 2005; 129:1825-1831. [PubMed: 16344051]

35. Cho JH, Kim HM, Ko GJ, et al. Old age and male sex are associated with increased risk of asymptomatic erosive esophagitis: analysis of data from local health examinations by the Korean National Health Insurance Corporation. J Gastroenterol Hepatol. 2011; 26:1034-1038. [PubMed: 21299618]

36. Nozu T, Komiyama H. Clinical characteristics of asymptomatic esophagitis. J Gastroenterol. 2008; 43:27-31. [PubMed: 18297432] 


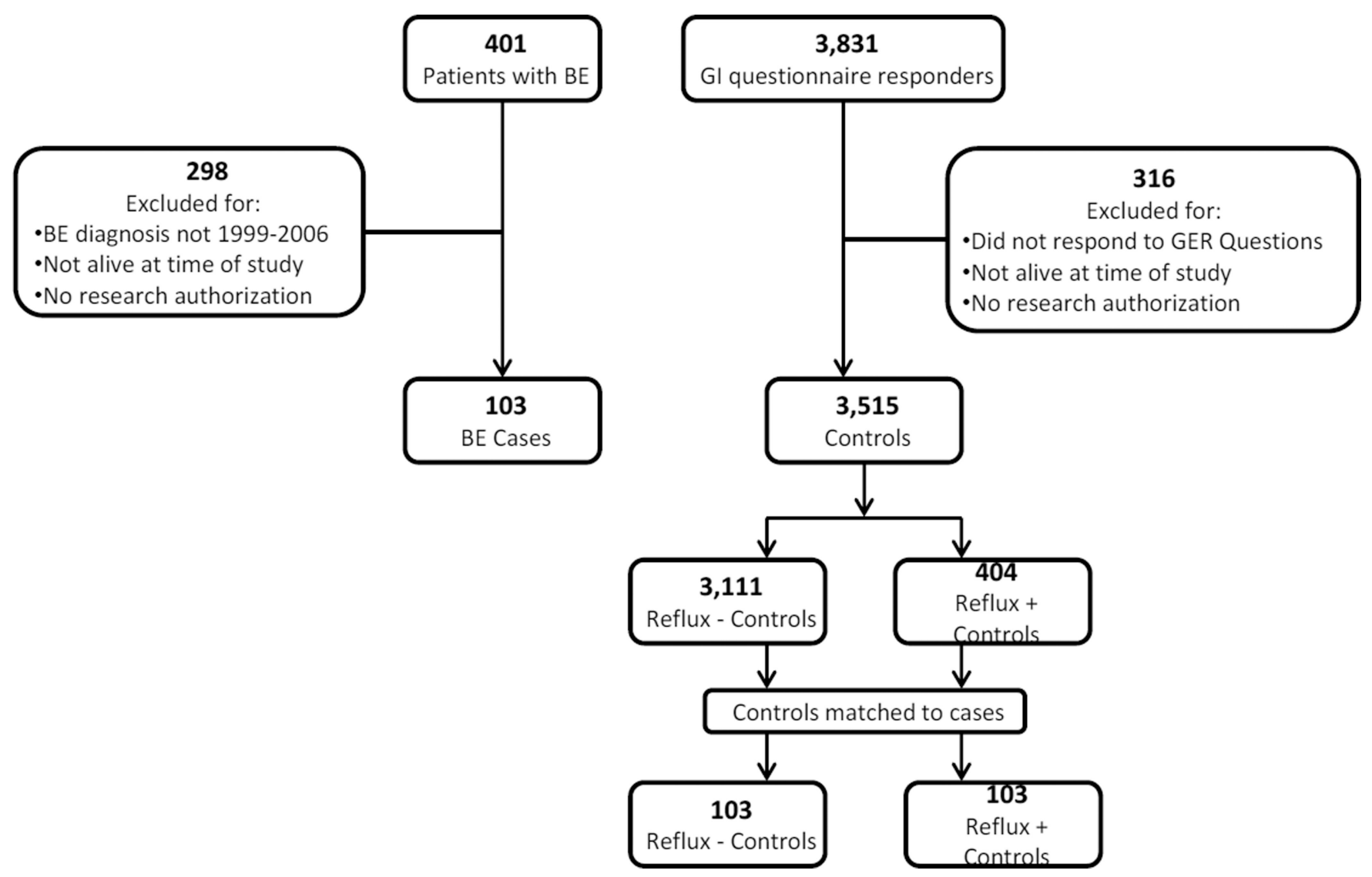

Figure 1.

Flowsheet describing the process of identification of cases and controls from previous cohorts. 


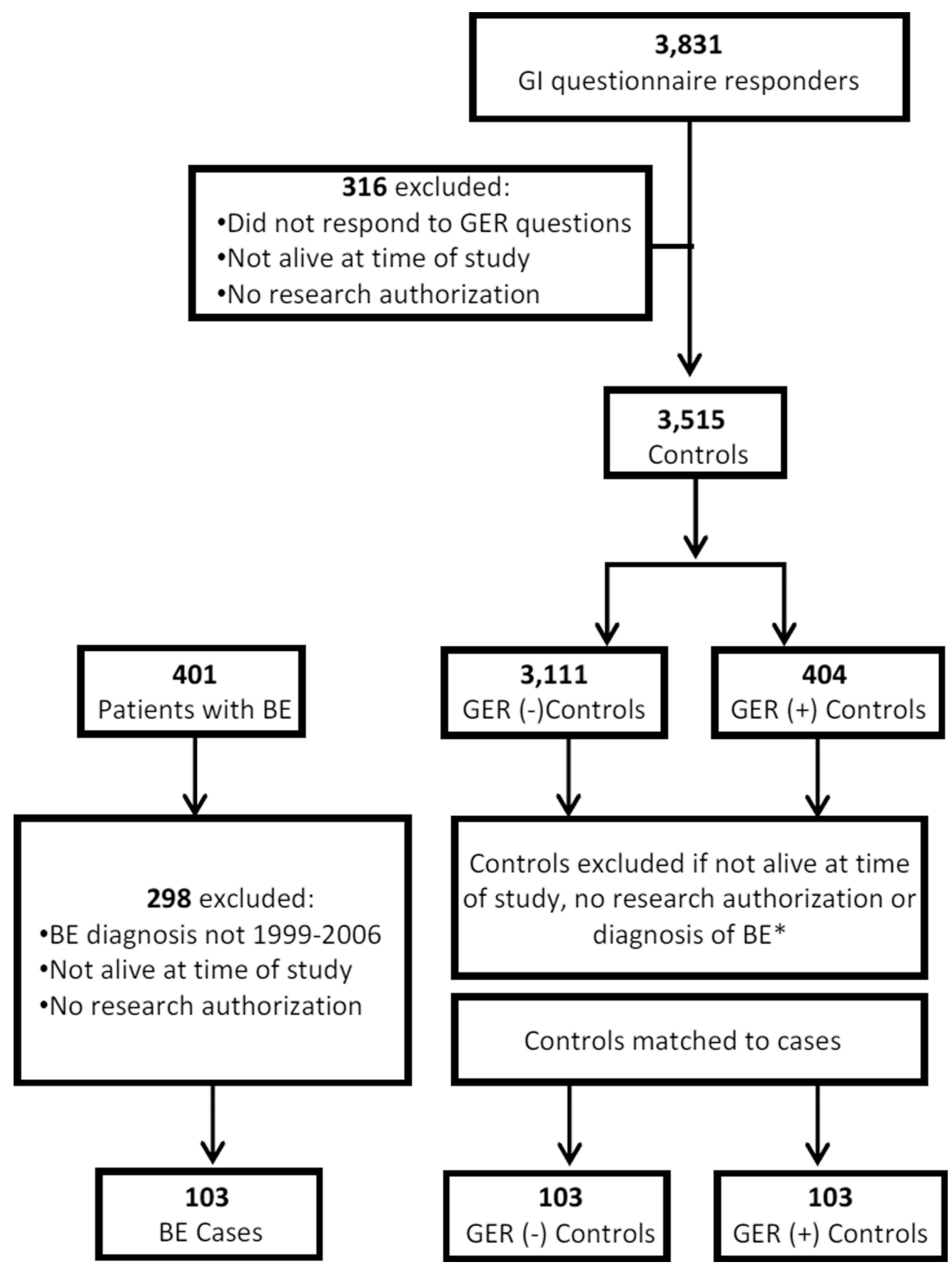

Figure 2.

Cases identified through the Rochester Epidemiology Project. Controls identified through a validated gastrointestinal questionnaire with focus on the frequency and nature of gastroesophageal reflux symptoms (heartburn, reflux). Reflux (GER (+)) positive controls defined by the presence of at least weekly heartburn and/or reflux symptoms. Reflux negative (GER (-)) controls defined by no reported heartburn or reflux symptoms. Cases and controls matched into triplets by age ( $\pm 10 \mathrm{yrs})$, sex and duration of follow-up as determined by date of first recorded medical encounter. $(*)$ a single reflux positive control was excluded due to diagnosis of BE. 


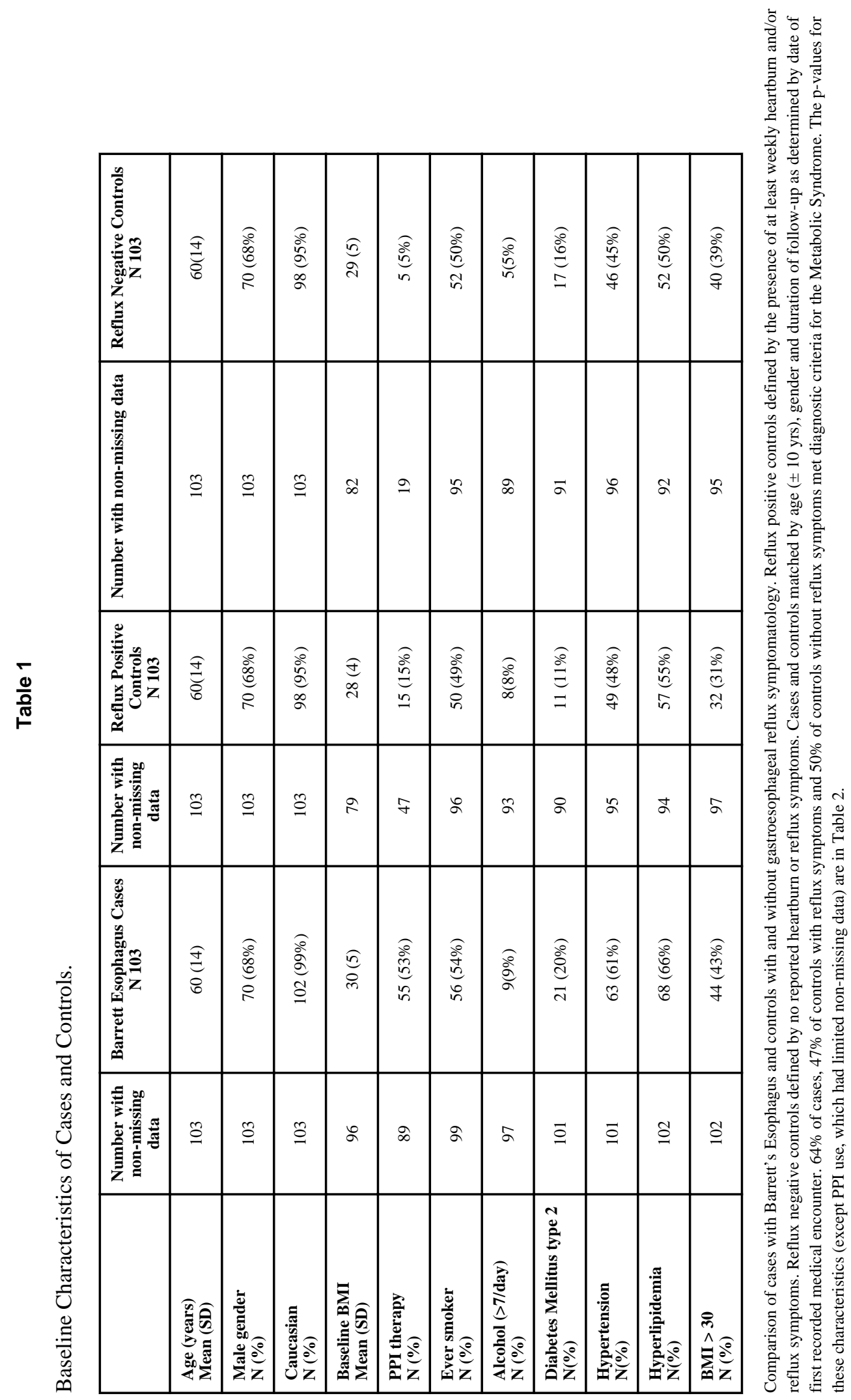

Mayo Clin Proc. Author manuscript; available in PMC 2014 February 01. 


\section{Table 2}

Univariate Models to Assess the Risk for Barrett's Esophagus: Metabolic syndrome (as a composite diagnosis), its Individual components and other risk factors for BE.

\begin{tabular}{|l|c|c|c|c|}
\hline & $\begin{array}{c}\text { Barrett's } \\
\text { Esophagus Cases } \\
\text { vs } \\
\text { Reflux Positive } \\
\text { Controls }\end{array}$ & & $\begin{array}{c}\text { Barrett's } \\
\text { Esophagus Cases } \\
\text { vs } \\
\text { Reflux Negative } \\
\text { Controls }\end{array}$ & \\
\hline & OR (95\% CI) & p-value & OR (95\% CI) & p-value \\
\hline Metabolic syndrome & $2.0(1.1,3.6)$ & 0.02 & $1.9(1.03,3.6)$ & 0.04 \\
\hline - BMI & $1.0(1.0,1.1)$ & 0.12 & $1.0(1.0,1.1)$ & 0.58 \\
\hline - Diabetes Mellitus type II & $1.9(0.8,4.4)$ & 0.15 & $1.1(0.6,2.3)$ & 0.72 \\
\hline - Hypertension & $1.5(0.8,2.7)$ & 0.22 & $2.4(1.2,4.8)$ & 0.02 \\
\hline - Hyperlipidemia & $1.4(0.8,2.7)$ & 0.26 & $1.7(0.9,3.3)$ & 0.11 \\
\hline Smoking-ever & $1.1(0.6,2.1)$ & 0.66 & $1.1(0.6,2.1)$ & 0.66 \\
\hline Alcohol intake (> 7 drinks/day) & $1.1(0.4,3.2)$ & 0.80 & $2.0(0.5,8.0)$ & 0.33 \\
\hline
\end{tabular}

Univariate analyses of Metabolic syndrome considered as a composite diagnosis and the individual factors used to define the Metabolic syndrome for the risk of Barrett's esophagus compared to controls with and without gastroesophageal reflux symptomatology. Reflux positive controls defined by the presence of at least weekly heartburn and/or reflux symptoms. Reflux negative controls defined by no reported heartburn or reflux symptoms. Analysis of risk associated with the Metabolic syndrome (composite diagnosis) based on 98 matched pairs in each model. BMI: body mass index 
Table 3

Multiple Variable Models to Assess the Risk for Barrett's Esophagus Using Individual Metabolic syndrome components.

\begin{tabular}{|l|c|c|c|c|}
\hline & $\begin{array}{c}\text { Barrett's Esophagus } \\
\text { Cases vs. Reflux } \\
\text { Positive Controls }\end{array}$ & & $\begin{array}{c}\text { Barrett's Esophagus } \\
\text { Cases vs } \\
\text { Reflux Negative } \\
\text { Controls }\end{array}$ & \\
\hline Risk Factors & OR (95\% CI) & p-value & OR (95\% CI) & p-value \\
\hline - BMI & $1.1(1.0,1.1)$ & 0.14 & $1.0(0.9,1.1)$ & 0.97 \\
\hline $\begin{array}{l}\text { - Diabetes Mellitus } \\
\text { type II }\end{array}$ & $1.2(0.5,3.1)$ & 0.73 & $0.9(0.4,2.0)$ & 0.71 \\
\hline - Hypertension & $1.4(0.6,3.1)$ & 0.41 & $2.4(1.0,5.6)$ & 0.05 \\
\hline - Hyperlipidemia & $1.1(0.5,2.6)$ & 0.75 & $1.3(0.6,2.7)$ & 0.56 \\
\hline
\end{tabular}

Multiple variable analyses of the individual factors used to define the Metabolic syndrome for the risk of Barrett's esophagus compared to controls with and without gastroesophageal reflux symptomatology. Reflux positive controls defined by the presence of at least weekly heartburn and/or reflux symptoms. Reflux negative controls defined by no reported heartburn or reflux symptoms. Analysis based on 81 matched pairs for the reflux positive control group and 83 matched pairs for reflux negative control group with complete data on each listed component of the Metabolic syndrome. BMI: body mass index 